\begin{tabular}{ll} 
PSS & PROCEEDNGS \\
\hline OF SCIENCE
\end{tabular}

Beyond renormalization in $D=4$ : an essay on nonlinear sigma model, massive $\mathrm{YM}$ and Electroweak Model

\title{
Daniele Bettinelli
}

Albert-Ludwigs-Universität Freiburg

E-mail: Daniele.Bettinelli@physik.uni-freiburg.de

\section{Ruggero Ferrari*}

CTP-MIT, Cambridge, MA

and

Università degli Studi di Milano \& INFN, Sez. di Milano

E-mail: ruggero.ferrari@mi.infn.it

\section{Andrea Quadri}

Albert-Ludwigs-Universität Freiburg

and

Università degli Studi di Milano \& INFN, Sez. di Milano

E-mail: andrea.quadri@mi.infn.it

We briefly outline the strategy of divergences removal in the Non Linear Sigma Model (NLSM), the Massive Yang-Mills (MYM) theory and the $S U(2) \otimes U(1)$ Electroweak Model (EW). By using a new Local Functional Equation (LFE) the subtraction of the divergences is performed on the basis of a criterion of symmetry, while preserving unitarity, locality of counterterms and predictivity of the models. 


\section{Introduction}

In power counting renormalizable theories there is a universally accepted rule, by which to every independent divergent one-particle-irreducible amplitude (1PI) one must associate a parameter in the tree-level action. This rule cannot be easily exported to any program of subtraction of infinities in nonrenormalizable theories. In fact, if this rule is used, the theory looses (in general) any predictivity and moreover the perturbative approach is unstable: for every new divergent 1PI amplitude emerging in the perturbative expansion, the whole series have to be updated from the beginning. Thus a novel procedure should be devised in order to make finite a nonrenormalizable theory. In this work we present a very promising strategy.

A common structure is present in the NLSM, in the MYM and in the Higgsless EW model. For $S U(2)$ one has the action structures: NLSM (Ref. [1]-[6])

$$
S_{N L S M}=\Lambda^{D-4} \frac{M^{2}}{4} \int d^{D} x \operatorname{Tr}\left\{\partial^{\mu} \Omega^{\dagger} \partial_{\mu} \Omega\right\}
$$

the Stuickelberg mass for YM (Ref. [7]-[8])

$$
S_{Y M} \sim \Lambda^{D-4} M^{2} \int d^{D} x \operatorname{Tr}\left\{\left[A_{\mu}-i \Omega \partial_{\mu} \Omega^{\dagger}\right]^{2}\right\}
$$

and EW (Ref. [9]-[11]) mass terms

$$
\begin{aligned}
& S_{E W} \sim \Lambda^{D-4} M^{2} \int d^{D} x\left(\operatorname{Tr}\left\{\left(g A_{\mu}-\frac{g^{\prime}}{2} \Omega \tau_{3} B_{\mu} \Omega^{\dagger}-i \Omega \partial_{\mu} \Omega^{\dagger}\right)^{2}\right\}\right. \\
&\left.+\frac{\kappa}{2}\left[\operatorname{Tr}\left\{g A_{\mu}-\frac{g^{\prime}}{2} \Omega \tau_{3} B_{\mu} \Omega^{\dagger}-i \Omega \partial_{\mu} \Omega^{\dagger} \tau_{3}\right\}\right]^{2}\right) .
\end{aligned}
$$

The $2 \times 2 \in S U(2)$ matrix may be parametrized by the real fields

$$
\Omega=\phi_{0}+i \tau_{i} \phi_{i}, \quad \phi_{0}=\sqrt{1-\vec{\phi}^{2}} .
$$

The constraint is implemented in the path integral measure

$$
\prod_{x} \mathscr{D}^{4} \phi(x) \theta\left(\phi_{0}\right) \delta\left(\vec{\phi}(x)^{2}+\phi_{0}^{2}(x)-1\right)=\prod_{x} \mathscr{D}^{3} \phi(x) \frac{2}{\sqrt{1-\vec{\phi}^{2}}}
$$


Vertexes carry second power of momenta, therefore already at one loop there is an infinite number of independent divergent amplitudes. Moreover it has been shown in the seventies and in the eighties that some divergences break (global) chiral invariance at the same order [12], [13], [14]. This effect seems to be a consequence of the non-trivial measure in the path integral. Thus we propose a new strategy [1]: abandon Hamiltonian formalism and do perturbation theory directly on the effective action functional $\Gamma$.

\section{The Local Functional Equation (LFE)}

The measure is invariant under "local left multiplication" transformations $\Omega \rightarrow U(\omega(x)) \Omega$

$$
\begin{aligned}
& \delta \phi_{0}=-\frac{\omega_{a}(x)}{2} \phi_{a} \\
& \delta \phi_{a}=\frac{\omega_{a}(x)}{2} \phi_{0}+\frac{\omega_{c}(x)}{2} \varepsilon_{a b c} \phi_{b} .
\end{aligned}
$$

We exploit this invariance and derive a new LFE. The invariance of the path integral functional under the substitution of the integration variables given by the above equations yields a local functional equation, since the parameters $\left(\omega_{a}(x)\right)$ are arbitrary functions.

Technical work to do: (i) find the algebra of operators closed under local left multiplication transformations by starting from the classical action, (ii) associate to every composite operator an external classical source (for subtraction strategy), (iii) write the LFE which follows from the invariance of the path integral measure.

Step (i)

This is simple in the NLSM. For this purpose one introduces a "gauge field"

$$
F_{\mu}=\frac{\tau_{a}}{2} F_{a \mu} \equiv i \Omega \partial_{\mu} \Omega^{\dagger} .
$$

Its field strength tensor is zero (it describes a scalar mode) and its transformation properties are those of a gauge field

$$
F_{\mu} \rightarrow U F_{\mu} U^{\dagger}+i U \partial_{\mu} U^{\dagger} .
$$

The classical action can be written

$$
S_{N L S M}=\Lambda^{D-4} \frac{M^{2}}{4} \int d^{D} x \operatorname{Tr}\left\{F_{\mu} F^{\mu}\right\} .
$$

Thus the closed set of operator is $\left\{\vec{\phi}, \phi_{0}, \vec{F}_{\mu}\right\}$ 


\section{Step (iii)}

Now we exploit the invariance of the path integral measure under local left multiplication $\left(\delta \phi_{a}=\right.$ $\left.\frac{\omega_{a}(x)}{2} \phi_{0}+\frac{\omega_{c}(x)}{2} \varepsilon_{a b c} \phi_{b}\right)$. We expand for small parameter $\vec{\omega}(x)$ and obtain the LFE $(\langle\cdots\rangle$ indicates the mean over the weighted paths )

$$
\begin{aligned}
& \int d^{D} x\left\langle\left( M_{D}^{2}(F-J)_{a \mu}\left(\varepsilon_{a b c} \omega_{c} F_{b}^{\mu}+\partial^{\mu} \omega_{a}\right)\right.\right. \\
& \left.\left.-\Lambda^{D-4} K_{0} \frac{\omega_{a}}{2} \phi_{a}+\phi_{0} K_{a} \frac{\omega_{a}}{2}+\varepsilon_{a b c} K_{a} \omega_{c} \phi_{b}\right)(x)\right\rangle=0,
\end{aligned}
$$

where

$$
M_{D}^{2} \equiv \Lambda^{D-4} M^{2}
$$

We will use the notation

$$
\mathscr{D}[X]_{a b}^{\mu}=\delta_{a b} \partial_{\mu}-\varepsilon_{a b c} X_{c \mu} .
$$

Thus for the effective action we get the local functional equation

$$
-\partial^{\mu} \frac{\delta \Gamma}{\delta J_{a}^{\mu}}+\varepsilon_{a b c} J_{c}^{\mu} \frac{\delta \Gamma}{\delta J_{b}^{\mu}}+\frac{\Lambda^{D-4}}{2} \phi_{a} K_{0}+\frac{1}{2 \Lambda^{D-4}} \frac{\delta \Gamma}{\delta K_{0}} \frac{\delta \Gamma}{\delta \phi_{a}}+\frac{1}{2} \varepsilon_{a b c} \phi_{c} \frac{\delta \Gamma}{\delta \phi_{b}}=0 .
$$

\section{Hierarchy}

The Spontaneous Breakdown of Symmetry is imposed by the condition

$$
\left.\frac{\delta \Gamma}{\delta K_{0}}\right|_{\text {field \&sources }=0}=1 .
$$

Then the LFE naturally induces a strong hierarchy structure among the 1PI irreducible amplitudes: all amplitudes involving the $\vec{\phi}$ fields (descendant) are known in terms of the amplitudes involving only the (ancestor) sources $\vec{J}_{\mu}, K_{0}$. For instance, if we differentiate the LFE with respect to $J_{a^{\prime}}^{v}(y)$ we get

$$
\frac{M_{D}^{2}}{2} \partial^{\mu} \frac{\delta^{2} \Gamma}{\delta J_{a}^{\mu}(x) \delta J_{a^{\prime}}^{v}(y)}+\frac{\delta^{2} \Gamma}{\delta \phi_{a}(x) \delta J_{a^{\prime}}^{v}(y)}+2 \delta_{a a^{\prime}} \partial_{x^{v}} \delta(x-y)=0
$$


where $I$ is the number of propagators, $N_{F}$ the number of external $F_{\mu}$ sources and $N_{K_{0}}$ those of $K_{0} . V_{j k}$ denotes the number of vertexes with $k \phi$-lines and $j$ derivatives. The superficial degree of divergence $\delta(G)$ for a graph can be bounded by using standard arguments. By removing $I$ from these two equations one gets

$$
\delta(G)=D n_{L}-2 n_{L}-\sum_{j, k}(2-j) V_{j k}-N_{F}-2 N_{K_{0}}+2 .
$$

The classical action has vertexes with $j \leq 2$, therefore it can be stated that

$$
\delta(G) \leq n_{L}(D-2)+2-N_{F}-2 N_{K_{0}} .
$$

For instance at $n_{L}=1$ the only ancestor divergent (independent) amplitudes are $(J-J),(J-J-J)$, $(J-J-J-J),\left(K_{0}-J-J\right),\left(K_{0}-K_{0}\right)$. The one-loop divergences of graphs where the descendant field appears $(\vec{\phi})$ are expressible all in terms of the ancestor divergences.

\section{Perturbative Expansion}

This is an Ansatz. We consider the generic dimension $D$ and we start with $\Gamma^{(0)}$. We read from it the value of the vertexes and construct $\Gamma^{(n)}$ for $n>0$. The connected amplitudes $W^{(n)}$ can then be obtained. Few questions are in order

1. Does $\Gamma^{(0)}$ obey the LFE? The answer is Yes, by construction.

2. Does $\Gamma^{(n)}, n>0$ obey the linearized LFE?

$$
\begin{aligned}
& \left(-\partial^{\mu} \frac{\delta}{\delta J_{a}^{\mu}}+\varepsilon_{a b c} J_{c}^{\mu} \frac{\delta}{\delta J_{b}^{\mu}}+\frac{1}{2 \Lambda^{D-4}} \frac{\delta \Gamma^{(0)}}{\delta \phi_{a}} \frac{\delta}{\delta K_{0}}+\frac{1}{2} \phi_{0} \frac{\delta}{\delta \phi_{a}}+\frac{1}{2} \varepsilon_{a b c} \phi_{c} \frac{\delta}{\delta \phi_{b}}\right) \Gamma^{(n)} \\
& +\sum_{j=1}^{n-1} \frac{1}{2 \Lambda^{D-4}} \frac{\delta \Gamma^{(j)}}{\delta \phi_{a}} \frac{\delta \Gamma^{(n-j)}}{\delta K_{0}}=0 .
\end{aligned}
$$

3. Assume that a symmetric subtraction procedure is given for the divergences in the limit $D=4$, how is the breaking of the above equation?

\subsection{Answers}

The answers to these questions are given in a compact form by the Quantum Action Principle

$$
\left(-\partial^{\mu} \frac{\delta}{\delta J_{a}^{\mu}}+\varepsilon_{a b c} J_{c}^{\mu} \frac{\delta}{\delta J_{b}^{\mu}}-\frac{\Lambda^{D-4}}{2} K_{0} \frac{\delta}{\delta K_{a}}+\frac{1}{2 \Lambda^{D-4}} K_{a} \frac{\delta}{\delta K_{0}}+\varepsilon_{a c b} K_{c} \frac{\delta}{\delta K_{b}}\right) Z
$$




\section{Subtraction Strategy}

Thus if the counterterms at order $n$ are missing, the linearized LFE is broken by the term

$$
\begin{aligned}
& \left(-\partial^{\mu} \frac{\delta}{\delta J_{a}^{\mu}}+\varepsilon_{a b c} J_{c}^{\mu} \frac{\delta}{\delta J_{b}^{\mu}}+\frac{1}{2 \Lambda^{D-4}} \frac{\delta \Gamma^{(0)}}{\delta \phi_{a}} \frac{\delta}{\delta K_{0}}\right. \\
& \left.+\frac{1}{2} \phi_{0} \frac{\delta}{\delta \phi_{a}}+\frac{1}{2} \varepsilon_{a b c} \phi_{c} \frac{\delta}{\delta \phi_{b}}\right) \Gamma^{(n)}=-\frac{1}{2 \Lambda^{D-4}} \sum_{j=1}^{n-1} \frac{\delta \hat{\Gamma}^{(j)}}{\delta K_{0}} \frac{\delta \hat{\Gamma}^{(n-j)}}{\delta \phi_{a}} .
\end{aligned}
$$

Notice that $\frac{1}{\Lambda^{D-4}} \frac{\delta \Gamma^{(0)}}{\delta \phi_{a}}$ is independent from $\Lambda^{D-4}$. Thus we use Laurent expansion on

$$
\Lambda^{-D+4} \Gamma^{(n)}
$$

to define the finite part and the counterterm $\Lambda^{-D+4} \hat{\Gamma}^{(n)}=-\left.\Lambda^{-D+4} \Gamma^{(n)}\right|_{\text {poles }}$.

The subtraction strategy works in this way: the multiplication by $\Lambda^{-D+4}$ removes the finite parts of the Laurent expansion of the non-homogeneous term. Therefore the subtraction of only the pole parts in the $\Lambda^{-D+4} \Gamma^{(n)}$ removes the non-homogeneous term and therefore the subtracted amplitude satisfies the linearized LFE. The subtracted poles are inserted as counterterms in the generic D effective action after a change of sign and a restoration of the $\Lambda^{D-4}$ factor.

\subsection{Organization of the Divergences}

The LFE is a powerful organizer of the divergences that WPC has classified. The full control can be obtained by finding the relevant local solutions of the linearized LFE

$$
\left(-\partial^{\mu} \frac{\delta}{\delta J_{a}^{\mu}}+\varepsilon_{a b c} J_{c}^{\mu} \frac{\delta}{\delta J_{b}^{\mu}}+\frac{1}{2 \Lambda^{D-4}} \frac{\delta \Gamma^{(0)}}{\delta \phi_{a}} \frac{\delta}{\delta K_{0}}+\frac{1}{2} \phi_{0} \frac{\delta}{\delta \phi_{a}}+\frac{1}{2} \varepsilon_{a b c} \phi_{c} \frac{\delta}{\delta \phi_{b}}\right) \Gamma^{(n)}\left[\vec{\phi}, \vec{J}_{\mu}, K_{0}\right]=0(7.3)
$$

Once the local solutions are known, the counterterms $\hat{\Gamma}^{(n)}$ can be written as linear superposition of them, where the coefficients are determined by the divergent ancestor amplitudes (finite in number because of the WPC). These local solutions can be easily constructed by using the technique of bleaching. We shortly describe this procedure. 
This suggests the bleaching

$$
\begin{aligned}
\mathfrak{J}_{\mu} & \equiv \Omega^{\dagger}\left(J_{\mu}-F_{\mu}\right) \Omega \\
\mathfrak{K}_{0} & \equiv \frac{K_{0}}{\phi_{0}}-\frac{M^{2}}{4}\left(J_{b}^{\mu}-F_{b}^{\mu}\right) \frac{\partial F_{b \mu}}{\partial \phi_{a}} \phi_{a}
\end{aligned}
$$

\subsection{Care in Bleaching}

Few facts about bleaching. i) The relations are invertible, ii) In the case of $\mathfrak{J}_{a \mu}$, bleaching is a kind of gauge transformation where the parameters are the $\vec{\phi}$ fields

$$
\begin{aligned}
& \mathfrak{J}_{\mu}=\Omega^{\dagger} J_{\mu} \Omega+i \Omega \partial_{\mu} \Omega \\
& \partial_{\mu} \mathfrak{J}_{v}=\Omega^{\dagger}\left(\partial_{\mu}+\Omega \partial_{\mu} \Omega^{\dagger}\right)\left(J_{v}-F_{V}\right) \Omega=\Omega^{\dagger} \mathscr{D}_{\mu}[F]\left(J_{v}-F_{V}\right) \Omega
\end{aligned}
$$

iii) the invariants can be constructed by using $\mathfrak{J}_{\mu}$ and $\mathfrak{K}_{0}$ and their space-time derivatives. Ancestor amplitudes do not depend explicitly from $\vec{\phi}$. We consider only those relevant for the one-loop divergences.

\subsection{The one-loop Invariants}

$$
\begin{aligned}
& \mathscr{I}_{1}=\int d^{D} x\left[D_{\mu}(F-J)_{v}\right]_{a}\left[D^{\mu}(F-J)^{v}\right]_{a}, \\
& \mathscr{I}_{2}=\int d^{D} x\left[D_{\mu}(F-J)^{\mu}\right]_{a}\left[D_{v}(F-J)^{v}\right]_{a}, \\
& \mathscr{I}_{3}=\int d^{D} x \varepsilon_{a b c}\left[D_{\mu}(F-J)_{v}\right]_{a}\left(F_{b}^{\mu}-J_{b}^{\mu}\right)\left(F_{c}^{v}-J_{c}^{v}\right), \\
& \mathscr{I}_{4}=\int d^{D} x\left(\frac{K_{0}}{\phi_{0}}+\frac{M^{2}}{4}\left[F_{b}^{\mu}-J_{b}^{\mu}\right] \frac{\partial F_{b \mu}}{\partial \phi_{a}} \phi_{a}\right)^{2} \\
& \mathscr{I}_{5}=\int d^{D} x\left(\frac{K_{0}}{\phi_{0}}+\frac{M^{2}}{4}\left[F_{b}^{\mu}-J_{b}^{\mu}\right] \frac{\partial F_{b \mu}}{\partial \phi_{a}} \phi_{a}\right)\left(F_{c}^{\mu}-J_{c}^{\mu}\right)^{2}, \\
& \mathscr{I}_{6}=\int d^{D} x\left(F_{a}^{\mu}-J_{a}^{\mu}\right)^{2}\left(F_{b}^{v}-J_{b}^{v}\right)^{2} \\
& \mathscr{I}_{7}=\int d^{D} x\left(F_{a}^{\mu}-J_{a}^{\mu}\right)\left(F_{a}^{v}-J_{a}^{v}\right)\left(F_{b \mu}-J_{b \mu}\right)\left(F_{b v}-J_{b v}\right),
\end{aligned}
$$


The right hand term is sterile: no descendant terms are generated. Explicit one-loop calculation gives [2]

$$
\begin{aligned}
& \Gamma^{(1)}=\frac{1}{D-4} \frac{\Lambda^{D-4}}{(4 \pi)^{2}}\left[-\frac{1}{12}\left(\mathscr{I}_{1}-\mathscr{I}_{2}-\mathscr{I}_{3}\right)+\frac{1}{48}\left(\mathscr{I}_{6}+2 \mathscr{I}_{7}\right)\right. \\
& \left.+\frac{3}{2} \frac{1}{M^{4}} \mathscr{I}_{4}+\frac{1}{2} \frac{1}{M^{2}} \mathscr{I}_{5}\right] .
\end{aligned}
$$

\section{The massive Yang-Mills}

In the massive Yang-Mills theory $\Omega$ enters in the mass term as in eq. (1.2) and describes the Goldstone bosons, that are here unphysical modes. Then it is important to ensure that the SlavnovTaylor Identity (STI) is valid in order to preserve unitarity. The LFE must be compatible with the STI. A suitable gauge-fixing term will help to achieve this result. The Landau gauge is the simplest, since the tadpole contributions can be neglected in most cases. The transformations to be considered are the local left $S U(2)_{L}$ and the global right $S U(2)_{R}$ on $\Omega$, the gauge fields $A_{\mu}$, the Faddeev-Popov fields $c, \bar{c}$. Few external sources are needed in order to describe the complete (under the $\left.S U(2)_{L} \otimes S U(2)_{R}\right)$ set of composite operators.

\subsection{Landau Gauge-fixing}

$$
\begin{gathered}
\Gamma^{(0)}=S_{Y M}+\frac{\Lambda^{D-4}}{g^{2}} \int d^{D} x\left(B_{a}\left(D^{\mu}[V]\left(A_{\mu}-V_{\mu}\right)\right)_{a}-\bar{c}_{a}\left(D^{\mu}[V] D_{\mu}[A] c\right)_{a}\right) \\
+\int d^{D} x\left(A_{a \mu}^{*} s A_{a}^{\mu}+\phi_{0}^{*} s \phi_{0}+\phi_{a}^{*} s \phi_{a}+c_{a}^{*} s c_{a}+K_{0} \phi_{0}\right) . \\
S_{Y M}=\frac{\Lambda^{(D-4)}}{g^{2}} \int d^{D} x\left(-\frac{1}{4} G_{a \mu \nu}[A] G_{a}^{\mu v}[A]+\frac{M^{2}}{2}\left(A_{a \mu}-F_{a \mu}\right)^{2}\right) . \\
\Omega=\frac{1}{v}\left(\phi_{0}+i \tau_{a} \phi_{a}\right), \quad \phi_{0}^{2}+\phi_{a}^{2}=v^{2}
\end{gathered}
$$

where $v$ is a parameter with dimension equal one. We stress that $v$ is not a parameter of the model, because it can can be removed by a rescaling of the fields $\vec{\phi}, \phi_{0}$. 


\section{MYM: the Local Functional Equation}

The procedure outlined for the NLSM can be repeated here for the MYM. The generating functional for the Green functions is given by a path integral which is invariant for a change of the integration variables under local left multiplication transformations. Thus one obtains a LFE

$$
\begin{aligned}
& \mathscr{W}(\Gamma) \equiv \int d^{D} x \alpha_{a}^{L}(x)\left(-\partial_{\mu} \frac{\delta \Gamma}{\delta V_{a \mu}}+\varepsilon_{a b c} V_{c \mu} \frac{\delta \Gamma}{\delta V_{b \mu}}-\partial_{\mu} \frac{\delta \Gamma}{\delta A_{a \mu}}\right. \\
& +\varepsilon_{a b c} A_{c \mu} \frac{\delta \Gamma}{\delta A_{b \mu}}+\varepsilon_{a b c} B_{c} \frac{\delta \Gamma}{\delta B_{b}}+\frac{1}{2} K_{0} \phi_{a}+\frac{1}{2} \frac{\delta \Gamma}{\delta K_{0}} \frac{\delta \Gamma}{\delta \phi_{a}} \\
& +\frac{1}{2} \varepsilon_{a b c} \phi_{c} \frac{\delta \Gamma}{\delta \phi_{b}}+\varepsilon_{a b c} \bar{c}_{c} \frac{\delta \Gamma}{\delta \bar{c}_{b}}+\varepsilon_{a b c} c_{c} \frac{\delta \Gamma}{\delta c_{b}} \\
& +\varepsilon_{a b c} A_{c \mu}^{*} \frac{\delta \Gamma}{\delta A_{b \mu}^{*}}+\varepsilon_{a b c} c_{c}^{*} \frac{\delta \Gamma}{\delta c_{b}^{*}}+\frac{1}{2} \phi_{0}^{*} \frac{\delta \Gamma}{\delta \phi_{a}^{*}} \\
& \left.+\frac{1}{2} \varepsilon_{a b c} \phi_{c}^{*} \frac{\delta \Gamma}{\delta \phi_{b}^{*}}-\frac{1}{2} \phi_{a}^{*} \frac{\delta \Gamma}{\delta \phi_{0}^{*}}\right)=0 .
\end{aligned}
$$

$\Gamma$ also obeys the Landau gauge equation

$$
\frac{\delta \Gamma}{\delta B_{a}}=\frac{\Lambda^{D-4}}{g^{2}} D^{\mu}[V]\left(A_{\mu}-V_{\mu}\right)_{a} .
$$

\section{Linearized Equations and Induced Transformations}

The structure of both STI and LFE is standard. Thus we can

1. Establish the full hierarchy (only the Goldstone bosons are descendant fields)

2. Confirm the validity of the WPC

3. Introduce the linearized STI and LFE

4. Extract from the linearized STI and LFE the generators of the transformations on the effective action $\Gamma$

5. Check that the generators stemming from STI commute with those from LFE 


\section{Example of the Subtraction Procedure}

In Ref. [8] we provided an explicit evaluation of the one-loop self-energy amplitude. We found that

1) The counterterms are local at the one-loop level.

2) The physical unitarity is valid.

The two-loop self-energy amplitudes have been considered from the point of view of the consistency. It has been argued that the subtraction scheme is consistent: i) the counterterms are local ii) physical unitarity is satisfied iii) the STI and LFE induced symmetry on $\Gamma$ is preserved.

\section{Conclusions}

The LFE for the NLSM, MYM and EW provides a strong tool to organize the divergences of the Feynman amplitudes and moreover hints for a consistent (i.e. symmetric and local) strategy for removing all the infinities. The steps in this procedure are

- Hierarchy

- WPC

- Minimal dimensional subtraction.

- Finite number of physical parameters

Moreover for massive YM, STI and Landau gauge equation guarantee

- Physical unitarity

- Consistency with LFE.

't Hooft-Feynman gauge is possible (many tadpole diagrams).

\section{Outlook and (some) open questions}

The future en gagement will be on 


\section{A. NOTE 1: LFE = Schwinger-Dyson equation?}

The Schwinger-Dyson equation in presence of a non trivial path integral measure is

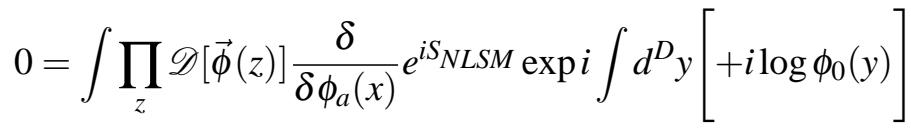

i.e.

$$
\left\langle\frac{\delta S_{N L S M}}{\delta \phi_{a}(x)}-i \frac{\phi_{a}}{\phi_{0}^{2}}\right\rangle=0
$$

Finally one gets

$$
\langle\frac{M_{D}^{2}}{\phi_{0}}(-\frac{1}{2} \partial^{\mu} F_{a \mu}+\underbrace{\varepsilon_{a b c} \square \phi_{b} \phi_{c}}) \underbrace{-i \frac{\phi_{a}}{\phi_{0}^{2}}}\rangle=0 .
$$

The under-braced terms are not present in the LFE. Moreover the overall factor $1 / \phi_{0}$ is also absent in the LFE. In fact the Schwinger-Dyson equation might coincide with the LFE only for trivial path integral measure (i.e. invariant under shift of the fields $\delta \phi_{a}=\alpha_{a} \in \mathfrak{R}$ ) [15].

\section{B. NOTE 2: LFE induced transformations}

One can formally introduce external currents by using the Legendre transform

$$
K_{a} \equiv-\frac{\delta}{\delta \phi_{a}} \Gamma^{(0)}=-\frac{\delta}{\delta \phi_{a}} S_{0}+\Lambda^{D-4} \frac{K_{0}}{\phi_{0}} \phi_{a},\left.\quad S_{0} \equiv \Gamma^{(0)}\right|_{K_{0}=0}
$$

Under the action

$$
\begin{aligned}
& \delta_{0} \equiv \int d^{D} x\left[\left(\partial^{\mu} \omega_{a}-\varepsilon_{a j i} J_{i}^{\mu} \omega_{j}\right) \frac{\delta}{\delta J_{a}^{\mu}}+\frac{1}{2 \Lambda^{D-4}} \omega_{a} \frac{\delta \Gamma^{(0)}}{\delta \phi_{a}} \frac{\delta}{\delta K_{0}}\right. \\
& \left.+\left(\frac{1}{2} \omega_{a} \phi_{0}+\frac{1}{2} \varepsilon_{a j k} \phi_{j} \omega_{k}\right) \frac{\delta}{\delta \phi_{a}}\right]
\end{aligned}
$$

we get 
and

$$
\delta_{0} K_{0}=\frac{1}{2 \Lambda^{D-4}} \omega_{a} \frac{\delta \Gamma^{(0)}}{\delta \phi_{a}} \frac{\delta}{\delta K_{0}} K_{0}=-\frac{1}{2 \Lambda^{D-4}} \omega_{a} K_{a} .
$$

Thus we get a bleached variable

$$
\begin{aligned}
& \mathfrak{K}_{0}=K_{0} \phi_{0}+\frac{1}{\Lambda^{D-4}} K_{a} \phi_{a} \\
& =\frac{K_{0}}{\phi_{0}}-\frac{1}{\Lambda^{D-4}} \phi_{a} \frac{\delta}{\delta \phi_{a}} S_{0}=\frac{K_{0}}{\phi_{0}}+\frac{M^{2}}{4}\left(F_{b}^{\mu}-J_{b}^{\mu}\right) \frac{\partial F_{b \mu}}{\partial \phi_{a}} \phi_{a}
\end{aligned}
$$

\section{NOTE 3: The Rosetta Stone}

We write the mapping for the paper [2] and the present work notation (denoted by a hat)

$$
m_{D}^{2}=m^{D-2}, \hat{\phi}_{0}=\frac{\phi_{0}}{m_{D}}, \hat{\phi}_{i}=\frac{g}{m_{D}} \phi_{i}, \hat{F}_{a \mu}=g F_{a \mu}, \hat{\Lambda}=m, \frac{\hat{\Lambda}}{\hat{M}}=g, \hat{J}_{a \mu}=-4 \frac{g}{m_{D}^{2}} J_{a \mu}
$$

With these mappings we get

$$
\begin{aligned}
& \hat{\mathscr{I}}_{1}=g^{2} \mathscr{I}_{1}, \quad \hat{\mathscr{I}}_{2}=g^{2} \mathscr{I}_{2}, \quad \hat{\mathscr{I}}_{3}=g^{3} \mathscr{I}_{3}, \quad \hat{\mathscr{I}}_{4}=\frac{m_{D}^{4}}{m^{4}} \mathscr{I}_{4}, \\
& \hat{\mathscr{I}}_{5}=g^{2} \frac{m_{D}^{2}}{m^{2}} \mathscr{I}_{5}, \quad \hat{\mathscr{I}}_{6}=g^{4} \mathscr{I}_{6}, \quad \hat{\mathscr{I}}_{7}=g^{4} \mathscr{I}_{7}
\end{aligned}
$$

Finally we get the counterterms

$$
\begin{aligned}
& \hat{\Gamma}^{(1)}=\frac{1}{D-4}\left[-\frac{1}{12} \frac{g^{2}}{(4 \pi)^{2}} \frac{m_{D}^{2}}{m^{2}}\left(\mathscr{I}_{1}-\mathscr{I}_{2}-g \mathscr{I}_{3}\right)\right. \\
& \left.+\frac{1}{(4 \pi)^{2}} \frac{g^{4}}{48} \frac{m_{D}^{2}}{m^{2}}\left(\mathscr{I}_{6}+2 \mathscr{I}_{7}\right)+\frac{1}{(4 \pi)^{2}} \frac{3}{2} \frac{g^{4}}{m^{2} m_{D}^{2}} \mathscr{I}_{4}+\frac{1}{(4 \pi)^{2}} \frac{1}{2} \frac{g^{4}}{m^{2}} \mathscr{I}_{5}\right] \\
& =\frac{1}{D-4}\left[-\frac{1}{12} \frac{1}{(4 \pi)^{2}} \frac{m_{D}^{2}}{m^{2}}\left(\hat{\mathscr{I}}_{1}-\hat{\mathscr{I}}_{2}-\hat{\mathscr{I}}_{3}\right)\right. \\
& \left.+\frac{1}{(4 \pi)^{2}} \frac{1}{48} \frac{m_{D}^{2}}{m^{2}}\left(\hat{\mathscr{I}}_{6}+2 \hat{\mathscr{I}}_{7}\right)+\frac{1}{(4 \pi)^{2}} \frac{3}{2} \frac{g^{4} m_{D}^{2}}{m^{6}} \hat{\mathscr{I}}_{4}+\frac{1}{(4 \pi)^{2}} \frac{1}{2} \frac{g^{2} m_{D}^{2}}{m^{4}} \hat{\mathscr{I}}_{5}\right] \\
& =\frac{1}{D-4} \frac{\Lambda^{D-4}}{(4 \pi)^{2}}\left[-\frac{1}{12}\left(\hat{\mathscr{I}}_{1}-\hat{\mathscr{I}}_{2}-\hat{\mathscr{I}}_{3}\right)+\frac{1}{48}\left(\hat{\mathscr{I}}_{6}+2 \hat{\mathscr{I}}_{7}\right) \frac{3}{2} \frac{1}{M^{4}} \hat{\mathscr{I}}_{4}+\frac{1}{2} \frac{1}{M^{2}} \hat{\mathscr{I}}_{5}\right]
\end{aligned}
$$


[7] D. Bettinelli, R. Ferrari and A. Quadri, Phys. Rev. D 77 (2008) 045021 [arXiv:0705.2339 [hep-th]].

[8] D. Bettinelli, R. Ferrari and A. Quadri, Phys. Rev. D 77 (2008) 105012 [arXiv:0709.0644 [hep-th]].

[9] D. Bettinelli, R. Ferrari and A. Quadri, Int. J. Mod. Phys. A 24 (2009) 2639 [arXiv:0807.3882 [hep-ph]].

[10] D. Bettinelli, R. Ferrari and A. Quadri, arXiv:0809.1994 [hep-th].

[11] D. Bettinelli, R. Ferrari and A. Quadri, Phys. Rev. D 79, 125028 (2009) [arXiv:0903.0281 [hep-th]].

[12] A. Salam and J. Strathdee, Phys. Rev. D 2, 2869 (1970).

D. G. Boulware, Ann. Phys. (N.Y.) 56, 140 (1970).

J. M. Charap, Phys. Rev. D 2, 1554 (1970).

J. M. Charap, Phys. Rev. D 3, 1998 (1971).

J. Honerkamp, Nucl. Phys. B 36, 130 (1972).

J. Honerkamp and K. Meetz, Phys. Rev. D 3, 1996 (1971).

I. S. Gerstein, R. Jackiw, S. Weinberg and B. W. Lee, Phys. Rev. D 3, 2486 (1971).

[13] L. Tătaru, Phys. Rev. D 12, 3351 (1975).

[14] T. Appelquist and C. W. Bernard, Phys. Rev. D 23, 425 (1981).

[15] R. Ferrari, “On the Renormalization of the Complex Scalar Free Field Theory,” arXiv:0907.0426 [hep-th].

[16] D. Bettinelli, R. Ferrari and A. Quadri, "The Electroweak Model based on the Nonlinearly Realized Gauge Group," In Proceedings of the International Workshop on QCD Green Functions, Confinement and Phenomenology. September 7-11, 2009, ECT* Trento, Italy. Eds. John M. Cornwall, Arlene C. Aguilar, Daniele Binosi and Joannis Papavassiliou. 